\title{
ERRATUM
}

\section{Erratum to: Synthesis of vinasse-dolomite nanocomposite biochar via a novel developed functionalization method to recover phosphate as a potential fertilizer substitute}

\author{
Nima Kamali ${ }^{1}$, Abdollah RashidiMehrabadi $(\bowtie){ }^{1}$, Maryam Mirabi ${ }^{1}$, Mohammad Ali Zahed ${ }^{2}$ \\ 1 Civil, Water and Environmental Engineering Faculty, Shahid Beheshti University A.C., Tehran, Iran \\ 2 Cell and Molecular Biology Department, Faculty of Biological Sciences, Kharazmi University, Tehran, Iran
}

(C) Higher Education Press 2021

Erratum to: Front. Environ. Sci. Eng. 2020, 14(4): 70

Due to the typesetting error, there are some errors in the Equation (4) in the manuscript, the correction is as follows, and we apologize to the reader.

$$
R_{\mathrm{L}}=\frac{1}{1+K_{\mathrm{L}} C_{0}}
$$

The online version of the original article can be found at https://doi.org/10.1007/s11783-020-1249-6

\footnotetext{
Corresponding author

E-mail: address: a_rashidi@sbu.ac.ir
} 